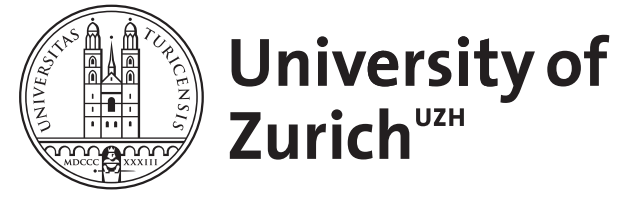

Zurich Open Repository and Archive

University of Zurich

University Library

Strickhofstrasse 39

CH-8057 Zurich

www.zora.uzh.ch

Year: 1994

\title{
Externe Handlungsgründe
}

Schaber, Peter

Posted at the Zurich Open Repository and Archive, University of Zurich

ZORA URL: https://doi.org/10.5167/uzh-80592

Book Section

Published Version

Originally published at:

Schaber, Peter (1994). Externe Handlungsgründe. In: Meggle, Georg; Wessels, Ulla. Analyomen 1 Proceedings of the 1st Conference "Perspectives in Analytical Philosophy". Berlin: De Gruyter, 842-849. 


\section{Contents}

Preface $\ldots \ldots \ldots \ldots \ldots \ldots \ldots \ldots \ldots \ldots \ldots \ldots, \mathrm{V}$

List of Contributors $\ldots \ldots \ldots \ldots \ldots \ldots \ldots \ldots \ldots \ldots \ldots \ldots$

Introduction

Georg Henrik von Wright

Analytische Philosophie - eine historisch-kritische Betrachtung ... 3

\section{Logic}

JaCQUeline Brunning

A Proposal for a Reconstruction of Peirce's 1870 Notation of Relatives 33

Christoph Fehige

The Limit Assumption in Deontic (and Prohairetic) Logic ..... 42

Bertram Kienzle

Ereignisse einer syrakusischen Nacht $\ldots \ldots \ldots \ldots \ldots \ldots \ldots \ldots$

Reinhard KLeinknecht

Theory of Descriptions and Truth-Set Semantics ........... 68

Helmut Linneweber-Lammerskitten

A Survey of the Derivability of Important Implicative Principles in Alternative Systems of Propositional Logic ............. 76

INGOLF MAX

Generalized Variable Functors Representing Paraconsistent Operators 88

Uwe Meixner

Nichttarskische Semantik der modalen Aussagenlogik ....... 98

UlRich MetsChL

Necessity and Provability . . . . . . . . . . . . . . . 103

UlRich NortManN

Does Aristotele's Modal Logic Rest on Metaphysical Assumptions? 115

Gerhard Schurz

Eine logische Analyse des Sein-Sollen-Problems $\ldots \ldots \ldots \ldots \ldots 126$ 
Peter Steinacker

Die epistemische Komponente nichtklassischer Funktoren.

Zwei Beispiele ......................... 135

Werner Stelzner

Relevanz, Konsistenz \& Entailment . . . . . . . . . . . . . 146

Horst Wessel

Alternative Logiken und empirische Wissenschaften

\section{Epistemology}

Stefan Gosepath

On the Rationality of Beliefs $\ldots \ldots \ldots \ldots \ldots \ldots \ldots \ldots \ldots, 179$

Michael Kober

Wittgenstein and Forms of Scepticism ............... 187

Dirk Koppelberg

Naturalistische Erkenntnistheorien und Probleme der Normativität 198

Franz von Kutschera

Zwischen Skepsis und Relativismus ................ 207

UlRich Majer

Ein konstruktiver Begriff der Wahrheit $\ldots \ldots \ldots \ldots \ldots \ldots \ldots .225$

Thomas Mormann

Cassirer's Problem and Geometrical Aspects of Epistemology .... 241

Sandra B. Rosenthal

Charles Peirce and the Issue of Foundations . . . . . . . . . . 251

Käthe Trettin

Logische Formalisierungen und Evidenz ................ 259

KLAus Volkert

Anschauliche Unmöglichkeit versus logische Unmöglichkeit zur erkenntnistheoretischen Diskussion über die nicht-euklidische Geometrie .............................. 266

\section{Philosophy of Science - Historical and Systematical}

Thomas BartelBorth

Wissenschaftlicher Realismus und approximative Theorien.

Zur Explikation des wissenschaftlichen Realismus ............ 
Andreas Bartels

Intertheoretische Begriffsrelationen und Referenzrahmen in der Physik ............................ 286

Adam Grobler

Justification of the Empirical Basis: The Popperian vs. the Inductivist

Conception of Rationality

Michael Heidelberger

Alternative Interpretationen der Repräsentationstheorie der Messung 310

Paul Hoyningen-Huene

Emergenz versus Reduktion

EkATERINI KaLERI

Zur strukturellen Analogie zwischen hermeneutischem Interpretieren und wissenschaftlichem Theoretisieren

AlFons KeupinK

Statistical Ambiguity and Inductive Inconsistencies . . . . . . . . 345

HaNs KRAML

Roger Bacon's Theory of the Rainbow as a Turning Point in the Pre-Galilean Theory of Science . . . . . . . . . . . . . . . 353

HaNs Scheibe

The Divorce Between the Sciences and the Humanities . . . . . . 362

Gerhard Terton

Methodologische Erklärungsmodelle aus heuristischer Sicht . . . . 379

Max URChs

Causal Priority. Towards a Logic of Event Causation . . . . . . . . 386

HANS WESTMEYER

Det strukturalistische Ansatz in der Theoretischen Psychologie . . . 397

IV. Philosophy of Language

C. Anthony Anderson

Degrees of Intentionality $\ldots \ldots \ldots \ldots \ldots \ldots \ldots \ldots \ldots \ldots$

ELKE BRENDEL

The Liar Paradox: An Extensional Alternative to the Situation

Semantics Approach . .....................

Filip Buekens

Keeping Track of Pierre's Mind. A Davidsonian Solution to Kripke's

Puzzle About Belief 
Christoph Jäger

Hybride singuläre Sinne und präsentische Propositionen $\ldots \ldots \ldots 444$ Matthias Kaufmann

Ockham und Davidson über die Wahrheit

M. Thomas Liske

Mögliche Welten bei David Lewis und bei Kripke

Ana Marostica

The Semiotic and the Semantic Conception of Truth

Felix Mühlhölzer \& Marianne Emödy

Über eine mögliche Inkonsistenz in Chomskys Auffassung von Sprachregeln ........................... 481

Albert Newen

How to Fix the Reference of 'that' in Demonstrative Utterances ... 493

Ulrich Pardey

Identität und Reflexivität . ...................... 509

Peter Philipp

PU $₫$ 293: Private und öffentliche Käfer $\ldots \ldots \ldots \ldots \ldots \ldots \ldots 520$

RICHARD RAATZSCH

„Die gemeinsame menschliche Handlungsweise“ (PU 206) . . . . . 529

ARTUR Rojszczak

Über die Korrespondenz von Tarskis Definition der Wahrheit ... . 539

EDMUND RUNGGALDIER

Referenz und „zeitliche Teile“ .................... 544

EIKE vON SAVIGNY

Stücke einer Definition des Wahrheitsbegriffs für bedeutungsvolle Äußerungen .................................. 550

Hans Julius SchNeider

Wie systematisch kann eine Theorie der Bedeutung sein? ....... 564

Oliver Scholz

Zum Status allgemeiner Verstehensprinizien $\ldots \ldots \ldots \ldots \ldots \ldots 574$

Klaus Wuttich

Bedingungen für den Sprechakt des Lügens $\ldots \ldots \ldots \ldots \ldots \ldots 58$

\section{Philosophy of Mind}

Antoni Gomila

Punctate Minds and Fodor's Theory of Content 605 
Michael ter Hark

Cognitive Science, Propositional Attitudes and the Debate Between Russell and Wittgenstein ......................6 612

Heinz-Dieter Heckmann

Can Personal Identity Be Analysed in Terms of Relations of

(Non-branching) Continuity? . . . . . . . . . . . . . . . . 618

GeERT KeIL

Is the Computational Metaphor of Mind Intentionalistic or

Naturalistic? . . . . . . . . . . . . . . . . . . . . . . . . . . . . . . . . . 629

Andreas Kemmerling

Mentale Repräsentationen - gibt es sie? . . . . . . . . . . . . . 640

Peter Lanz

Funktionalismus und sensorisches Bewußtsein .............6 648

Georg Meggle

Zukünftige Dienstage ....................... 660

Thomas Metzinger

Subjectivity and Mental Representation .............. 668

Audun $\varnothing_{\text {FSTI }}$

Searle, Leibniz and „The First Person“. A Note on the Epilogue of Intentionality . . . . . . . . . . . . . . . . . . . . . . . 682

SVEN ROSENKRANZ

A Review of Eccle's Arguments for Dualist-Interactionism . . . . . 689

Katia Saporiti

Fodors naturalistischer Begriff der Bedeutung . . . . . . . . . . . . 695

VI. Practical Philosophy

Antonella Corradini

Abtreibung und das Prinzip der Doppelwirkung . . . . . . . . . . 707

Rafael Ferber

Moral Judgements as Descriptions of Institutional Facts . . . . . . 719

Martina Herrmann

Wie beschafft man sich moralische Intuitionen? . . . . . . . . . 730

Helmut F. Kaplan

Ethik, Leid und Mitleid . . . . . . . . . . . . . . . . . . . . . 737

Matthias Kettrer

„Geltungsansprüche“ 
HANS LENK

Zwischen Metaphysik und normativen Interpretationskonstrukten die Wiederkehr praktischer Fragen in der analytischen Philosophie

Christoph Lumer

Was ist eine triftige Moralbegründung?

Julian Nida-Rümelin

Ethischer Kognitivismus ohne Intuitionen

Klaus Peter Rippe

Artenschutz als Problem der Praktischen Ethik

Beate Rössler

Quotierung als moralisches Problem .

ECKARD RoLF

Emotionen und Handlungen

Peter Schaber

Externe Handlungsgründe

Sabine Thürmel

Ethische Aspekte der virtuellen Realität

Jean-Claude Wolf

Utilitarismus, Verantwortung und kriminelle Versuche

VII. Miscellanea

WINFRIED Franzen

„Die wahre und einzige Methode der Philosophie ist also die analytische ...". Der junge Herder über die Philosophie und ihr Verhältnis zur Sprache

Dietfried Gerhardus

Die Rolle von Probe und Etikett in Goodmans Theorie der Exemplifikation .

Christian Kanzian

Der Begriff "Koinzidenz" in der Mereologie

HaRALD KöHL

Selbstbestimmung und Verzweiflung

WINFRIED LÖFFLER

Modale Versionen des ontologischen Arguments für die Existenz Gottes 
Gebhard Löhr

Kann der Glaube an Gott die Frage nach dem Sinn des Lebens beantworten? .............................. 916

Kuno LóRENZ

Was ist der Mensch? - Auch eine Frage der analytischen Philosophie 927 Jaroslav Peregrin

Formalisation of Language as a Means of Philosophical Analysis .. 939

ROLF W. PUSTER

Sprachanalytisches Argumentieren bei John Locke .......... 946

Antonio Zilhao

Ludwig Wittgenstein and Edmund Husserl ............. 956

Appendices

Appendix One

Satzung der Gesellschaft für Analytiscbe Pbilosophie (GAP) ...... 967

Appendix Two

Georg Meggle: Bericht des Präsidenten vor der 1. Mitgliederversammlung der Gesellschaft für Analytische Philosophie (GAP) am 10.10 .1991 in Saarbrücken . . . . . . . . . . . . . . . . . 972

Ergänzungen und Nachträge vom 10. 8. 1992 ............ 977

Index $\ldots \ldots \ldots \ldots \ldots \ldots \ldots \ldots \ldots \ldots \ldots \ldots . . .679$ 
Bereitgestellt von | UZH Hauptbibliothek / Zentralbibliothek Zürich Angemeldet Heruntergeladen am | 30.11.17 14:27 